\title{
Repurposing Nonantifungal Approved Drugs for Synergistic Targeting of Fungal Pathogens
}

\author{
Cindy Vallières, Nishant Singh, Cameron Alexander, and Simon V. Avery* \\ Cite This: ACS Infect. Dis. 2020, 6, 2950-2958 \\ Read Online
}

ABSTRACT: With the spread of drug resistance, new antimicrobials are urgently needed. Here, we set out to tackle this problem by high-throughput exploration for novel antifungal synergies among combinations of approved, nonantifungal drugs; a novel strategy exploiting the potential of alternative targets, low chemicals usage and low development risk. We screened the fungal pathogen Candida albicans by combining a small panel of nonantifungal drugs (all in current use for other clinical applications) with 1280 compounds from an approved drug library. Screens at sublethal concentrations of the antibiotic paromomycin (PM), the antimalarial primaquine (PQ), or the anti-inflammatory drug ibuprofen (IF) revealed a total of 17 potential strong, synergistic interactions with the library compounds. Susceptibility testing with the most promising combinations corroborated marked synergies [fractional inhibitory concentration (FIC) indices $\leq 0.5$ ] between $\mathrm{PM}+\beta$-escin, $\mathrm{PQ}+$

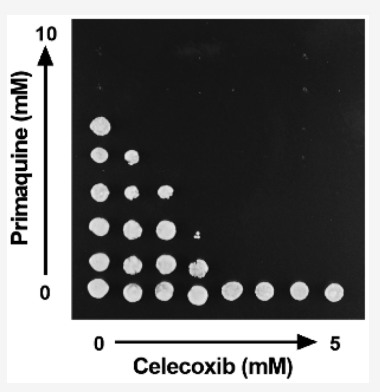
celecoxib, and IF + pentamidine, reducing the MICs of PM, PQ and IF in C. albicans by $>64-, 16-$, and 8 -fold, respectively. Paromomycin $+\beta$-escin and $\mathrm{PQ}+$ celecoxib were effective also against $C$. albicans biofilms, azole-resistant clinical isolates, and other fungal pathogens. Actions were specific, as no synergistic effect was observed in mammalian cells. Mode of action was investigated for one of the combinations, revealing that PM $+\beta$-escin synergistically increase the error-rate of mRNA translation and suggesting a different molecular target to current antifungals. The study unveils the potential of the described combinatorial strategy in enabling acceleration of drug-repurposing discovery for combatting fungal pathogens.

KEYWORDS: Candida glabrata, Cryptococcus neoformans, Aspergillus fumigatus, candidiasis, fungicide, mistranslation

$\mathrm{F}$ ungal pathogens have emerged as a leading cause of human mortality. Recent estimates suggest that invasive fungal infections are associated with mortality rates $\sim 50 \%$ in hospitalized patients and are killing over 1.6 million people annually, more than major diseases such as malaria and breast cancer. ${ }^{1,2}$ The striking impact on human health is due partly to a limited number of approved antifungal drugs and the emergence of resistance to those current treatments. ${ }^{2}$ The lack of antifungals can be ascribed at least partly to the conservation of eukaryotic gene functions between fungi and humans, making drug discovery challenging. ${ }^{3}$ Echinocandins are the most recent class of antifungals, but it has been 14 years since the last echinocandin, anidulafungin, was introduced to the market. $^{4}$

Combinatorial inhibition of fungal growth provides an attractive strategy to help control fungal diseases. Drug combinations can allow use of decreased doses of each compound compared to monotherapy, potentially lowering costs and toxicity, ${ }^{5-7}$ especially where the agents act synergistically, i.e., with a combined effect greater than that expected from the individual drug effects. For example, a synergistic combination consisting of amphotericin B and 5flucytosine is used to treat cryptococcosis. ${ }^{8}$

The repurposing of existing, approved drugs for alternative uses as antifungals has emerged as a further approach to facilitate drug development. ${ }^{3,9-11}$ A key advantage is that information on toxicology and pharmacokinetics from preclinical and clinical trials is already available, lowering regulatory barriers, development time, and cost. Recent studies identified drugs in FDA-approved libraries that could enhance efficacy of current antifungal drugs, such as fluconazole, caspofungin, and amphotericin B, against Candida and Cryptococcus spp. ${ }^{9,10}$ Chemical genetic analysis showed that these chemicals belonged to two classes of compounds, which either perturbed membrane permeability or inhibited sphingolipid biosynthesis. ${ }^{9,10}$ However, resistance is already well documented for the antifungal drugs cited above, with potential for cross-resistance also to agents (possibly synergistic) that act at a related target, undermining the potential long-term value of combinations involving these agents. Therefore, combinations of agents where neither is a current antifungal could have particular value as a future antifungal strategy. The use of chemosensitizers (i.e., compounds that further sensitize cells to the effects of a bioactive agent) to potentiate the effect of a nonantifungal drug has shown promising results. ${ }^{12}$ Here, we developed a

Received: June 10, 2020

Published: November 3, 2020

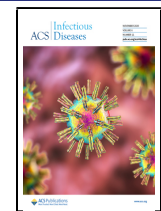


A
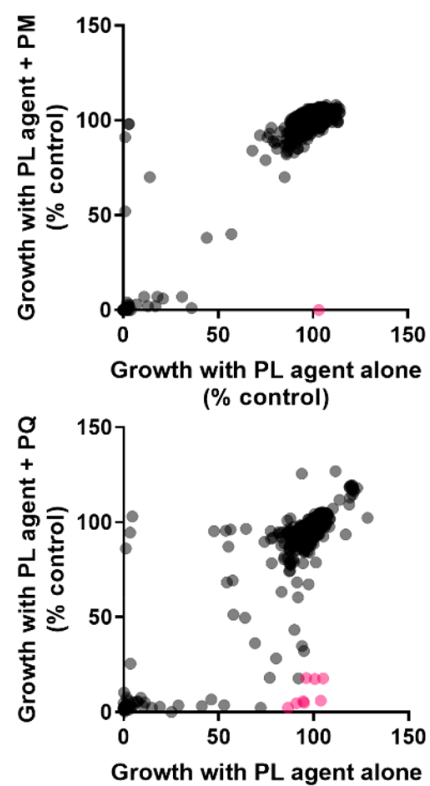

(\% control)

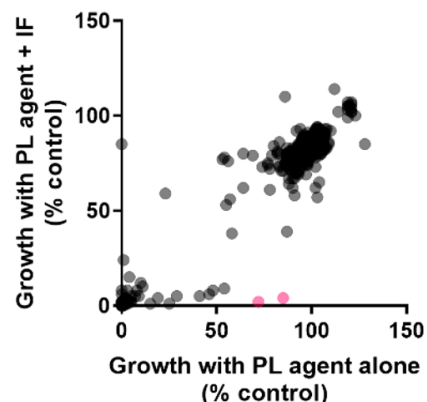

B
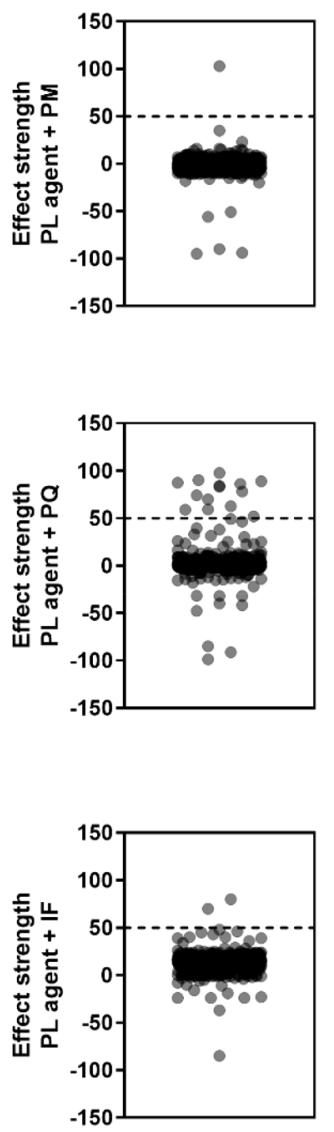

C

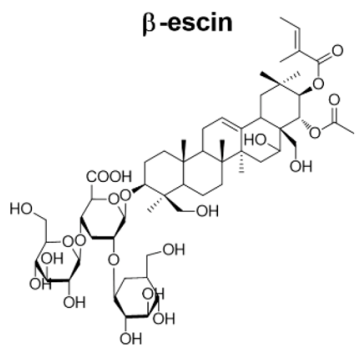<smiles>Cc1ccc(-c2cc(C(F)(F)F)nn2-c2ccc(S(N)(=O)=O)cc2)cc1</smiles>

Pentamidine isethionate<smiles>N=C(N)c1ccc(OCCCCCOc2ccc(C(=N)N)cc2)cc1</smiles>

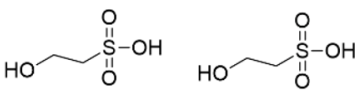

Figure 1. Chemical screening data for compounds that potentiate three nonantifungals in C. albicans. PL compounds were supplied at $100 \mu \mathrm{M}$. (A) The scatterplots show the normalized growth of $C$. albicans for each PL compound in the absence $(x$ axis $)$ and presence of either PM, PQ, or IF $(y$ axis). The data are means from duplicate screens. The full screen data are in Table S1. (B) Effect strength of the different combinations; those that showed an effect strength $>50$ are colored in pink in A. (C) The names and structures of library compounds giving the greatest effect strength from each screen.

screening strategy to identify compounds from large libraries of approved drugs that could act in synergy with common nonantifungal drugs. For the latter, to establish proof-ofprinciple we used the aminoglycoside antibiotic paromomycin (PM), the antimalarial primaquine (PQ), and the nonsteroidal anti-inflammatory drug ibuprofen (IF). We report characterization of new drug combinations which synergistically and selectively target key fungal pathogens, including via novel mode of action. The results indicate a promising alternative strategy for targeting fungal pathogens by high-throughput exploration of combinatorial drug repurposing. By excluding current antifungal therapeutics, application of such an approach should also reduce selection for extant resistance, prolonging longevity of current antifungals.

\section{RESULTS}

Compounds from the Approved Drug Library Potentiate Action of Three Nonantifungal Drugs against C. albicans. A screen was performed against C. albicans with the Prestwick library (PL) of FDA-/EMEAapproved drugs in the absence and presence of subinhibitory concentrations of a small panel of exemplar nonantifungal drugs: paromomycin, $\mathrm{PM}$; primaquine, $\mathrm{PQ}_{i}$ ibuprofen, IF
(Figure 1A). The latter three drugs are themselves approved and used widely for different applications in humans. In addition, they have different modes of action, so broadening the scope for finding diverse chemical interactions in the screens. We also know from previous studies that those three drugs exhibit some antifungal activity but at concentrations, when supplied alone, not normally suitable for therapeutic purposes. ${ }^{13-15}$ The three agents are therefore ideal candidates for finding new combination-synergies that could enable therapeutic applications. Library compounds were supplied at $100 \mu \mathrm{M}$, the maximum concentration attainable while ensuring that the added volumes of drug stock solutions (in DMSO) did not exceed $2 \%$ of final volume. At this concentration, 90\% of the compounds had minimal growth effects when supplied alone (i.e., less than $20 \%$ reduction of growth) (Figure 1A; Table S1); therefore, this concentration was suitable for identifying strong enhancers of the three compounds of interest. Effect strength was calculated for each combination (see Materials and Methods) and those with an effect strength $>50$ were considered as combinations of interest, i.e., where the difference between \% growth with the PL compound alone and the combination was $>50$. From the 1280 PL compounds screened, 1, 14, and 2 showed an effect strength $>50$ when 


\section{A}

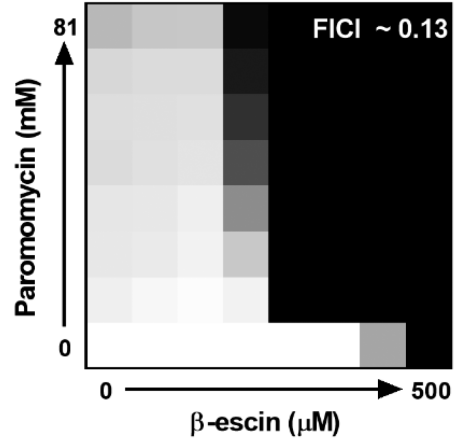

B

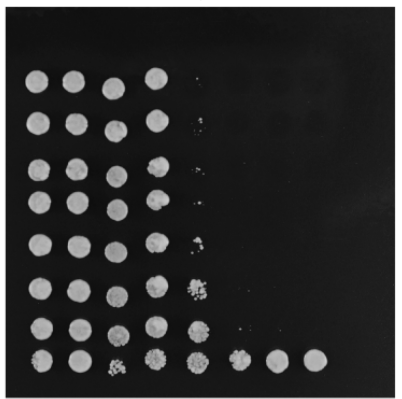

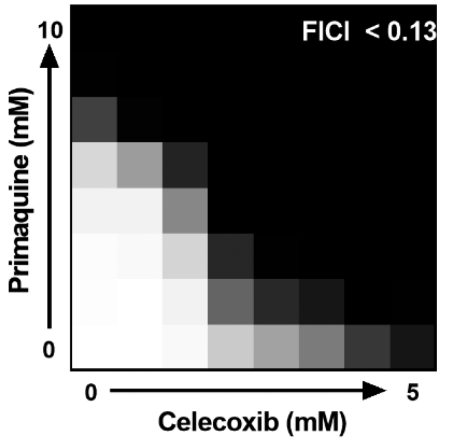

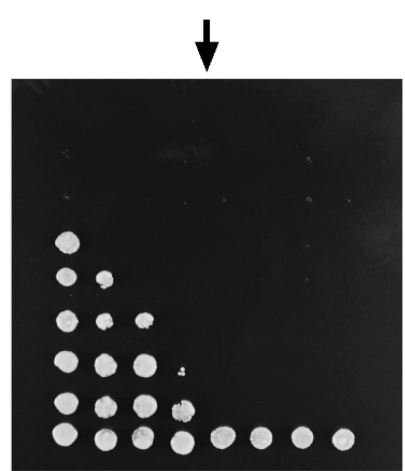

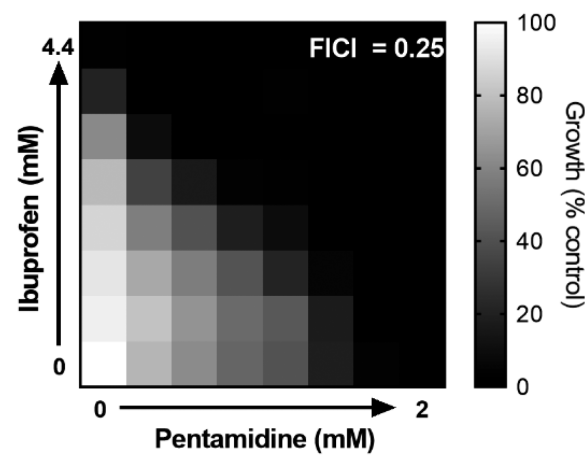

Pentamidine (mM)

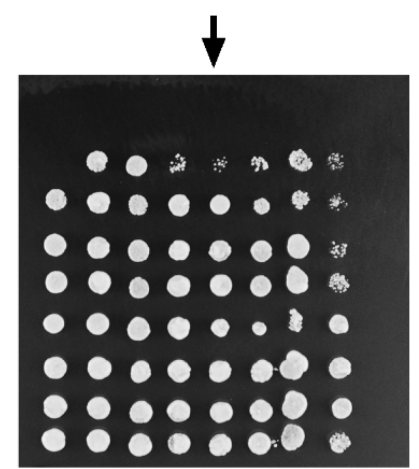

Figure 2. Lead combinations from the screens inhibit C. albicans synergistically. (A) Checkerboard assays of drug-drug interactions. Two-fold serial dilutions of the PL compounds were combined with 2 -fold dilutions of the three nonantifungal drugs. Growth $\left(\mathrm{OD}_{600}\right)$ was measured after 24 $\mathrm{h}$ and values obtained with agents expressed as percentages relative to the minus-drug controls. Data are means from at least three independent experiments. FICI, fractional inhibitory concentration index. (B) Cidal and static drug interactions. After $24 \mathrm{~h}$, cells from the checkerboard assays were spotted onto YPD agar without drugs and incubated for $24 \mathrm{~h}$ at $30{ }^{\circ} \mathrm{C}$ before plates were photographed. The images are representative of three independent experiments.

Table 1. Library Compound Chemical Interactions with the Nonantifungals in C. albicans

\begin{tabular}{|c|c|c|c|c|c|c|}
\hline & & \multicolumn{4}{|c|}{ planktonic cells of indicated strains } & \multirow{2}{*}{$\begin{array}{c}\text { biofilm } \\
\text { SC5314 }\end{array}$} \\
\hline & & SC5314 & J942148 & $\mathrm{J} 980280$ & SCS1192299X & \\
\hline \multirow[t]{5}{*}{ Paromomycin $(\mathrm{PM})$ and $\beta$-escin $(\beta \mathrm{E})$} & MIC PM (mM) & $>81.2$ & $>81.2$ & 1.27 & 1.27 & $>81.2$ \\
\hline & CIC PM (mM) & 1.27 & 2.54 & 0.32 & 0.32 & 20.3 \\
\hline & $\mathrm{MIC} \beta \mathrm{E}(\mu \mathrm{M})$ & 500 & $>500$ & 500 & 500 & $>500$ \\
\hline & $\mathrm{CIC} \beta \mathrm{E}(\mu \mathrm{M})$ & 62.5 & 62.5 & 125 & 125 & 125 \\
\hline & FICI & $\sim 0.13^{a}$ & $<0.08$ & 0.5 & 0.5 & $<0.25$ \\
\hline \multirow[t]{5}{*}{ Primaquine (PQ) and Celecoxib (CC) } & MIC PQ (mM) & 5 & 2.5 & 2.5 & 2.5 & 5 \\
\hline & $\mathrm{CIC} P Q(\mathrm{mM})$ & 0.31 & 0.63 & 0.16 & 0.31 & 1.25 \\
\hline & MIC CC (mM) & $>5$ & $>5$ & 2.5 & 2.5 & $>5$ \\
\hline & CIC CC (mM) & 0.63 & 1.25 & 0.31 & 0.31 & 1.25 \\
\hline & FICI & $0.06-0.13$ & $0.25-0.38$ & 0.19 & 0.5 & $0.25-0.38$ \\
\hline \multirow[t]{5}{*}{ Ibuprofen (IF) and Pentamidine (PD) } & MIC IF (mM) & 4.36 & 4.36 & 2.18 & 2.18 & $>4.36$ \\
\hline & CIC IF (mM) & 0.55 & 0.55 & 0.55 & 0.27 & 1.09 \\
\hline & MIC PD $(\mu \mathrm{M})$ & 1000 & 250 & 31.25 & 62.5 & 1000 \\
\hline & CIC PD $(\mu \mathrm{M})$ & 125 & 31.25 & 0.98 & 15.63 & 1000 \\
\hline & FICI & 0.25 & 0.25 & 0.28 & 0.38 & $1-1.13$ \\
\hline
\end{tabular}

${ }^{a}$ Drug interactions in planktonic cells and $24 \mathrm{~h}$ old biofilms were determined by checkerboard assays (see Materials and Methods). MIC, minimum inhibitory concentration. CIC, combination inhibitory concentration, the lowest inhibitory concentration of the agent achieved when in the combination. FICI, fractional inhibitory concentration index.

combined with $\mathrm{PM}, \mathrm{PQ}$ and $\mathrm{IF}$, respectively; these also showed less than $30 \%$ reduction of growth when supplied alone (Figure 1A,B; Table S1). The most promising library compound (i.e., giving the highest effect strength) for each of the three nonantifungal drugs was selected for further tests, i.e., $\beta$-escin (for PM), celecoxib (PQ), and pentamidine (IF) (Figure 1C).
The Drug Combinations Have Synergistic, Fungicidal, and Antibiofilm Actions in C. albicans. Checkerboard analysis was performed with $C$. albicans to corroborate synergistic interactions for the most promising drug combinations identified from the screens. Each of these three combinations was found to give synergistic inhibition of C. albicans growth. The combination decreased the minimum 
Table 2. Library Compound Chemical Interactions with the Nonantifungals in Several Fungal Pathogens and in Mammalian Cells

\begin{tabular}{|c|c|c|c|c|c|}
\hline & & Aspergillus fumigatus & Candida glabrata & Cryptococcus neoformans & mammalian cells \\
\hline \multirow[t]{5}{*}{ Paromomycin $(\mathrm{PM})$ and $\beta$-escin $(\beta \mathrm{E})$} & MIC PM (mM) & $>81.2$ & 10.2 & 0.16 & 81.2 \\
\hline & CIC PM (mM) & 40.6 & 2.54 & - & 40.6 \\
\hline & $\operatorname{MIC} \beta \mathrm{E}(\mu \mathrm{M})$ & $>500$ & 250 & $>500$ & 40 \\
\hline & $\mathrm{CIC} \beta \mathrm{E}(\mu \mathrm{M})$ & 125 & 62.5 & - & 10 \\
\hline & FICI & $<0.38^{a}$ & 0.5 & - & 0.75 \\
\hline \multirow[t]{5}{*}{ Primaquine (PQ) and Celecoxib (CC) } & MIC PQ (mM) & $>10$ & 10 & 0.63 & 0.1 \\
\hline & CIC PQ (mM) & 5 & 1.25 & 0.08 & 0.1 \\
\hline & MIC CC (mM) & $>5$ & $>5$ & 2.5 & 0.125 \\
\hline & CIC CC (mM) & 0.16 & 0.31 & 0.63 & 0.125 \\
\hline & FICI & $<0.27$ & $0.13-0.16$ & 0.38 & 2 \\
\hline
\end{tabular}

${ }^{a}$ Drug interactions were determined by checkerboard assays (see Materials and Methods). MIC, minimum inhibitory concentration. CIC, combination inhibitory concentration, the lowest inhibitory concentration of the agent achieved when in the combination. FICI, fractional inhibitory concentration index.

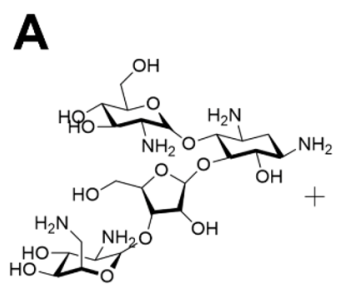

Paromomycin

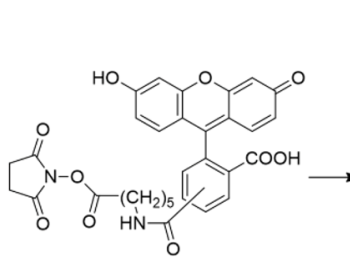

SFX

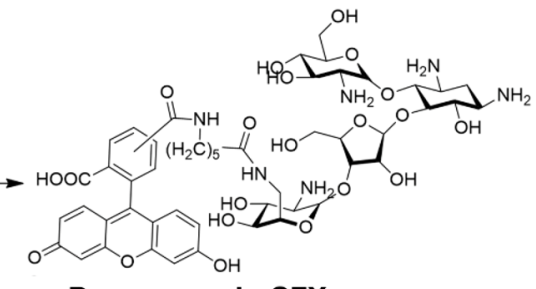

Paromomycin-SFX (major product)

B
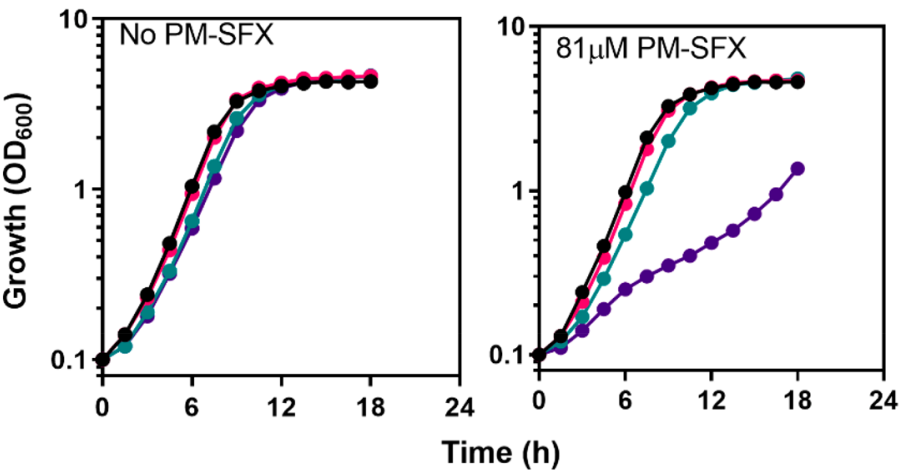

- no escin

$\rightarrow 25 \mu \mathrm{M} \beta$-escin

$\rightarrow 37.5 \mu \mathrm{M} \beta$-escin

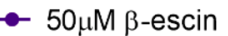

C

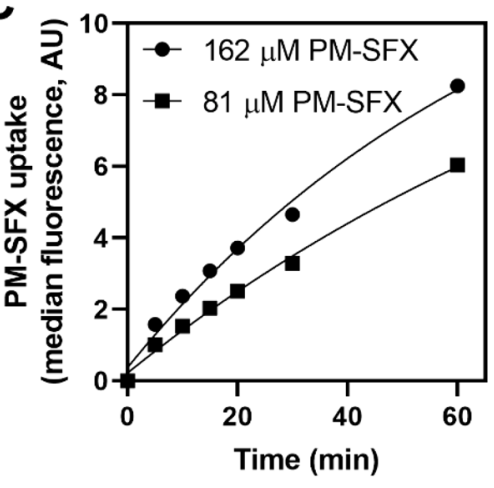

D

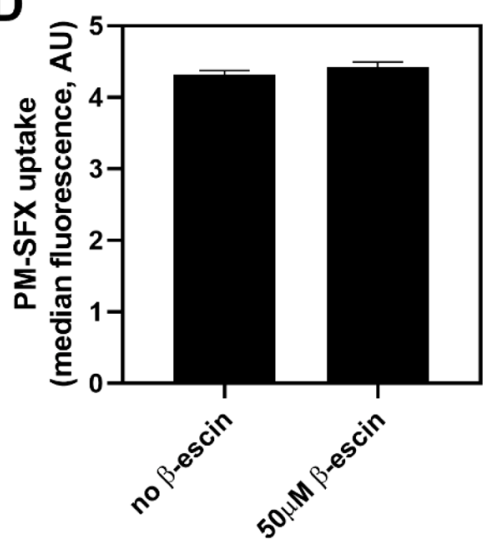

Figure 3. $\beta$-Escin does not increase PM-SFX uptake. (A) Synthesis of paromomycin-SFX. (B) Determination of inhibitory drug concentrations: $S$. cerevisiae was cultured in YPD with agents supplied at the specified concentrations. (C) Determination of PM-SFX uptake kinetics: Cells were incubated with PM-SFX at the indicated concentrations, and cellular PM-SFX determined at intervals with flow cytometry. Values shown are after subtraction of autofluorescence determined in cells incubated without PM-SFX. (D) Test of $\beta$-escin effect on PM-SFX uptake: S. cerevisiae was incubated for $20 \mathrm{~min}$ with $81 \mu \mathrm{M}$ PM-SFX in combination or not with $50 \mu \mathrm{M} \beta$-escin. AU, arbitrary units. All values are means \pm SEM from three independent determinations. 

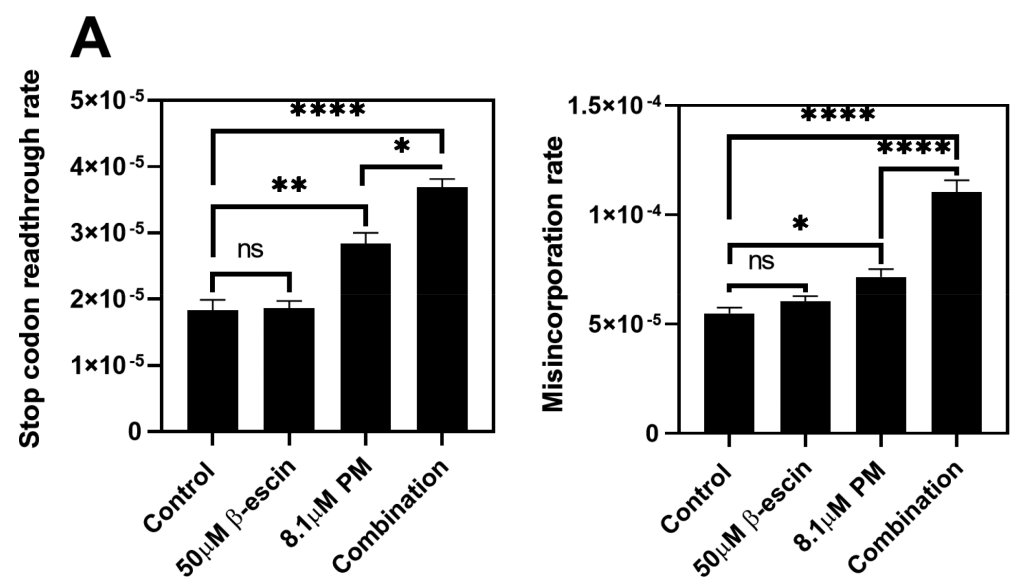

B

Figure 4. Paromomycin and $\beta$-escin synergistically increase mistranslation rate. (A) S. cerevisiae transformed with a dual-luciferase plasmid encoding the Renilla and firefly luciferases separated by a UAA stop codon (left) or containing a His $245 \rightarrow \operatorname{Arg} 245$ mutation in the firefly luciferase gene (right) were exposed to $50 \mu \mathrm{M} \beta$-escin or $8.1 \mu \mathrm{M} \mathrm{PM}$ alone or in combination for $16 \mathrm{~h}$ before determination of the two luciferase activities. The values are means \pm SEM from at least three independent experiments. ${ }^{*} p \leq 0.05$, ${ }^{*} p \leq 0.01, * * * * p \leq 0.0001$ according to multiple comparisons (Tukey's test) by one-way ANOVA; ns, not significant. Values were normalized for incidental variation in assay sensitivity between replicate experiments performed on different days. (B) S. cerevisiae containing a plasmid encoding a RFP and a nonfunctional Tyr66 $\rightarrow$ His66 GFP were treated with $30 \mu \mathrm{M} \beta$-escin or 20.3 or $40.6 \mu \mathrm{M} \mathrm{PM}$, alone or in combination for $16 \mathrm{~h}$ before cell fluorescence was measured by flow cytometry. Cells expressing GFP are shown as percentages of total cells. The values are means \pm SEM from three independent experiments. $* * p \leq$ $0.01, * * * * \leq 0.0001$ according to multiple comparisons (Tukey's test) by two-way ANOVA.

inhibitory concentrations (MICs) of the individual agents by $>64$-fold for PM, 16-fold for PQ and 8-fold for IF, with fractional inhibitory concentration indices (FICI; see Materials and Methods) of $\sim 0.13$ for $\mathrm{PM}+\beta$-escin, between 0.06 and 0.13 for $\mathrm{PQ}+$ celecoxib, and 0.25 for IF + pentamidine (a combination is considered synergistic when the FICI is $\leq 0.5$ ) (Figure 2A and Table 1).

Resistance among clinical fungal isolates has eroded the efficacy of current antifungals such as azoles, but resistance is typically less common against fungicidal than fungistatic compounds. ${ }^{16}$ The present data indicated that $\mathrm{PM}+\beta$-escin and $P Q+$ celecoxib are fungicidal, as $C$. albicans cells showing growth inhibition in the checkboard assays proved unable to form colonies when recovered to the control medium (Figure 2B). In contrast, IF + pentamidine was fungistatic.

Candida albicans has the ability to form drug-resistant biofilms, associated with high mortality rates in infected, immunocompromised patients. ${ }^{17}$ We tested the impact of the combinations against $24 \mathrm{~h}$ old biofilm structures formed in 96well microtiter plates. Biofilm metabolic activity was synergistically decreased with the combinations $\mathrm{PM}+\beta$-escin and PQ+ celecoxib, albeit with slightly higher FICI values compared to planktonic C. albicans SC5314 (Table 1). However, no synergistic effect (FICI > 0.5) was observed with IF + pentamidine, suggesting a different interaction only for this particular combination when treating biofilms compared to planktonic cells. Whereas we tested preformed biofilms, the fact that planktonic cells are affected by the combinations means that the formation of biofilms should also be inhibited (while we have no reason to expect that a particular step in the formation of biofilms should specifically be targeted by these combinations, e.g., adherence).

The Library Compounds Enhance the Effects of Paromomycin, Ibuprofen, and Primaquine in AzoleResistant C. albicans Isolates and in Other Fungal Pathogens. We evaluated the activity of the combinations against three azole-resistant clinical isolates. ${ }^{18}$ Certain of the clinical isolates showed differences in sensitivity to individual agents compared with the laboratory strain C. albicans SC5314. Moreover, as in the SC5314 strain, synergies were observed with each of the test combinations for all of the azole-resistant isolates (FICI $\leq 0.5)$ (Table 1$)$.

To indicate the spectrum of synergy against other fungal pathogens, the most promising combinations $\mathrm{PM}+\beta$-escin and $\mathrm{PQ}+$ celecoxib (both were fungicidal and active against biofilms), were tested against other key fungal species. It was not possible to determine high MICs for certain drugs and species, as the amounts of solvent added would have been inhibitory. This precluded estimation of FICI for the PM $+\beta$ escin combination in Cryptococcus neoformans. Nevertheless, synergistic inhibition by this combination could be demonstrated on the growth of Aspergillus fumigatus and Candida glabrata, and synergy of the PQ + celecoxib combination was evident against all three pathogens (Table 2). The strongest effect for $\mathrm{PM}+\beta$-escin was obtained against $A$. fumigatus (FICI < 0.38). The strongest synergy for PQ + celecoxib was with C. glabrata (FICI 0.13-0.16), giving striking reductions of the MICs when in combination (8-fold for PQ and $>16$-fold for celecoxib). FIC indices were lower for both combinations with C. albicans (Table 1). However, C. glabrata and C. neoformans were more sensitive to PM and $\beta$-escin, and $P Q$ and celecoxib, respectively, than C. albicans.

To assess specificity of synergy for the fungi, we also tested mammalian cells. The synergy appeared to be specific to the fungi as no synergy was detectable with NIH 3T3 cells, which showed FICI indices of 0.75 for $\mathrm{PM}+\beta$-escin and 2.0 for PQ+ celecoxib (Table 2). We also tested the combination $\mathrm{PM}+\beta$ escin against TE671 cells using subinhibitory concentrations of each drug. No significant difference in cell viability was observed between the cells treated with $\beta$-escin alone and the cells treated with the combination (Figure S2) supporting the absence of combinatorial cytotoxicity.

The $\beta$-Escin and Paromomycin Combination Promotes Synergistic Loss of Translation Fidelity. $\beta$-Escin is the major active component in extracts of horse chestnut seeds and has been reported to show certain antifungal proper- 
ties. $^{19,20}$ The compound interacts with sterol molecules creating pores in the cell membrane. ${ }^{21}$ Paromomycin is known to cause mRNA mistranslation. Previously, drug combinations involving PM have been reported to increase further the rate of translation error. ${ }^{13,22}$ We hypothesized that the $\mathrm{PM}+\beta$-escin mode of synergistic action could arise from $\beta$-escin allowing increased cellular entry of the aminoglycoside, leading to an increase in the error-rate of mRNA translation. To test this hypothesis, PM was tagged with the fluorescent probe SFX, a fluorescein analogue with a seven carbon spacer to ensure retention of fluorescence following conjugation (Figures 3A, S1). The addition of the SFX label to the drug did not affect the antifungal interaction, as PM-SFX still acted in synergy with $\beta$-escin on yeast growth (Figure 3B); Saccharomyces cerevisiae was used for these experiments as a range of mistranslation-assay tools are available with this model yeast (we first confirmed $\beta$-escin synergy with non-SFX labeled $\mathrm{PM}$ in S. cerevisiae). Yeast cells were treated with $81 \mu \mathrm{M}$ PMSFX alone or in combination with $50 \mu \mathrm{M}$ of $\beta$-escin for $20 \mathrm{~min}$ (Figure 3C) before cellular SFX fluorescence was measured by flow cytometry. No significant increase in PM-SFX uptake was observed in the presence of $\beta$-escin treatment (Figure 3D), suggesting elevated PM uptake was not responsible for the synergy observed between these compounds.

To test whether synergistic growth inhibition was reflected by synergistic mistranslation, we used quantitative assays of mistranslation. These included tests for readthrough of a UAA stop codon positioned between Renilla and firefly luciferase ORFs, or for amino acid misincorporation in firefly luciferase. The PM $+\beta$-escin combination increased the rate of stop codon readthrough and missense error significantly compared to both of the individual drug effects, with a stronger effect on missense than stop codon readthrough (Figure 4A). We also measured misincorporation rate in a strain expressing a nonfunctional GFP (Tyr66 $\rightarrow$ His66), constructed as part of this study. Significant restoration of cellular GFP fluorescence was observed when the cells were treated with the drug combination compared to no drug, or either drug alone (Figure 4B). The data suggest that $\beta$-escin synergistically potentiates the action of PM on translation fidelity and this effect is not due to increased PM uptake.

\section{DISCUSSION}

This study proposes an alternative combinatorial strategy for combatting fungal pathogens, by high-throughput exploration for novel antifungal synergies among pairs of approved drugs not currently useful for treating fungal infections and with diverse modes of action. We show that the approach successfully enables discovery of new, potent synergies. This strategy opens a potential treasure-trove of novel antifungal treatment-options, by exploiting the principle of drug synergy in a drug repurposing context.

High-throughput combinatorial antifungal screens have previously included at least one existing antifungal drug. For instance, by combining six known antifungals and fungicides with more than 3600 compounds in fungal species including C. albicans, a diverse range of chemical-chemical interactions were recently described. Eighteen compounds increased the susceptibility of a wild type strain of $C$. albicans to fluconazole. ${ }^{10}$ However, only three were synergistic with the azole against fluconazole-resistant clinical isolates, ${ }^{10}$ suggesting a limitation in using available antifungals for chemical screening as it is likely that existing resistance diminishes the effectiveness of the combinations. With increased recrudescence of fungal isolates resistant to the current arsenal of antifungals, new drugs with new mechanisms of action are needed. Nonantifungal drugs could therefore be repositioned as novel antifungal agents. Numerous drugs may affect fungal growth, but usually only at concentrations so high as to exclude them from therapeutic use for this purpose. One approach to achieving inhibition at lower doses is by using chemosensitizers. Chemosensitizing compounds possess antifungal activity, but at insufficient potency to serve alone. Their main modes of action are disruption of the fungal stress response, destabilization of the structural integrity of cellular and vacuolar membranes or stimulation of reactive oxygen species production making cells more sensitive to the effects of treatment. ${ }^{23}$ Bithionol, which is known to target antioxidant defense, has proven effective in combination with pro-oxidant chemosensitizers against the fungal pathogen A. fumigatus. ${ }^{12}$ Another strategy is by exploiting synergies between pairs of drugs; such synergies may arise where two active agents target any common process but by different mechanisms or pathways. ${ }^{13,22,24,25}$ A novel aspect of the present work is that we selected three nonantifungal approved drugs (i.e., paromomycin, primaquine, and ibuprofen) with distinct modes of action, to search for novel antifungal synergies in combinations with 1280 FDA-/EMEA-approved drugs. Prior approval of these off-patent agents are features that can help expedite development toward clinical application of potential hits.

Among 17 synergies identified from $\sim 3840$ interactions tested, three were selected for further study. $\beta$-Escin enhanced the effect of PM, producing a striking $>64$-fold decrease of the MIC of PM, the strongest decrease observed in this study. Further checkerboard assays over lower concentration ranges of the antibiotic showed that the MIC of PM could be decreased even further: to $812 \mu \mathrm{M}$ and $\sim 49 \mu \mathrm{M}$ when combined with $62.5 \mu \mathrm{M}$ and $125 \mu \mathrm{M} \beta$-escin, respectively (both subinhibitory concentrations) (Figure S3). The MIC range of PM in E. coli is from 3 to $208 \mu \mathrm{M}(0.002-0.128 \mathrm{mg}$ $\left.\mathrm{mL}^{-1}\right){ }^{26}$ The current dosing of PM in humans ranges from topical creams for cutaneous action through to intramuscular injections for systemic therapy and, for the latter, doses of 20 $\mathrm{mg}^{-1} \mathrm{~kg}^{-1}$ day $^{-1}$ have been employed, resulting in plasma concentrations of $\sim 10 \mu \mathrm{g} \mathrm{mL}^{-1}$. ${ }^{27}$ This corresponds to PM concentrations of $\sim 20 \mu \mathrm{M}$, and this level of drug in the plasma is retained for several hours. Therefore, the effective paromomycin concentrations when in combination are within the range that could be considered for (antifungal) therapeutic use, although a full human pharmacokinetics study of both drugs in combination would be required to establish the definitive therapeutic doses needed for synergism. We showed that the two drugs synergistically increase the rate of error in mRNA translation. Protein synthesis is an essential process and therefore can be an important drug target. The $\beta$-escin mechanism of action in this combination remains unclear, as it did not cause increased PM uptake. Recent studies indicated that $\beta$-escin has mistranslation (readthrough) activity of its own in mammalian cells. ${ }^{28,29}$

Another combination of interest characterized here comprised the antimalarial primaquine and the nonsteroidal anti-inflammatory drug (NSAID) celecoxib. It has been shown that PQ can target key iron-sulfur cluster proteins in yeast, such as aconitase, required for the tricarboxylic acid (TCA) cycle. ${ }^{15}$ Interestingly, the celecoxib derivative AR-12 was found 
to inhibit fungal acetyl-COA synthetase in vitro and has activity against a range of pathogenic yeasts and molds. ${ }^{30,31}$ Acetyl coenzyme A is a crucial metabolite for fungal metabolism including in the TCA cycle, suggesting one potential target of the synergy between $\mathrm{PQ}$ and celecoxib. Another NSAID cyclooxygenase inhibitor, ibuprofen, was a subject of this study. As ibuprofen targets mitochondria ${ }^{32}$ its synergistic inhibition of fungal growth when combined with the antibiotic pentamidine is not unexpected, as pentamidine is known to inhibit translation in yeast mitochondria. ${ }^{33}$

A decreased risk of resistance emergence against synergistic drug combinations should be marked when agents have fungicidal rather than fungistatic actions. ${ }^{16,34,35}$ Paromomycin $+\beta$-escin and primaquine + celecoxib combinations described here had fungicidal activity. Furthermore, these also showed efficacy against $C$. albicans biofilms, which typically have elevated resistance to antifungals. ${ }^{17}$ As well as being effective against a standard laboratory strain of $C$. albicans, these combinations also synergistically inhibited growth of azoleresistant clinical isolates and of other major fungal pathogens (A.fumigatus, C. glabrata, and C. neoformans), indicating broad spectrum actions of the type that can be advantageous in clinical use. Importantly, synergy was not recapitulated in mammalian cells, suggesting specificity for the fungi and consistent with the possibility of therapeutic applications.

The high-throughput strategy developed here successfully identified several novel antifungal synergies and could help bypass current antifungal resistances, from screens that were by no means exhaustive. It suggests that a much wider potential arsenal of antifungal activities are attainable with this approach. This is important considering the urgent, unmet need for new therapeutic antifungal options.

\section{MATERIALS AND METHODS}

Strains, Culture, and Maintenance. Pathogenic organisms used in this study were the yeasts Candida albicans SC5314 and the azole-resistant isolates J942148, J980280, and SCS1192299X (kindly provided by Carol Munro and Donna MacCallum, University of Aberdeen, UK), Cryptococcus neoformans 1841, Candida glabrata BG2, and Aspergillus fumigatus CBS 144.89. Mode of action studies were performed with Saccharomyces cerevisiae BY4743 (MATa/ $\alpha$ his $3 \Delta 1 /$ his $3 \Delta 1$ leu $2 \Delta 0 /$ leu $2 \Delta 0$ LYS2/lys $2 \Delta 0$ met $15 \Delta 0 / M E T 15$ ura $3 \Delta 0 /$ ura $3 \Delta 0)$. The fungi were maintained and grown in either YPD medium [2\% peptone (Oxoid), 1\% yeast extract (Oxoid), 2\% D-glucose], YNB medium $[0.69 \%$ yeast nitrogen base without amino acids (Formedium), 2\% D-glucose, supplemented with amino acids or nucleobases as appropriate for plasmids selection], ${ }^{36}$ potato dextrose agar or broth [PDA (Oxoid) or PDB (Sigma)], or RPMI $1640+2 \%$ glucose medium (Sigma). Where necessary, media were solidified with $1.5 \%$ agar (Sigma).

High-Throughput Screening. The Prestwick Chemical Library (PL) (Prestwick Chemical) was screened with drugs supplied at a final concentration of $100 \mu \mathrm{M}$ in the presence or absence of either $812 \mu \mathrm{M}\left(500 \mu \mathrm{g} \mathrm{mL}{ }^{-1}\right)$ paromomycin sulfate, $750 \mu \mathrm{M}\left(342 \mu \mathrm{g} \mathrm{mL} L^{-1}\right)$ primaquine biphosphate, or $436 \mu \mathrm{M}\left(90 \mu \mathrm{g} \mathrm{mL}^{-1}\right)$ ibuprofen (Sigma). (Among the PL drugs that gave synergies from the screens, $100 \mu \mathrm{M}$ corresponds to $113 \mu \mathrm{g} \mathrm{mL}^{-1} \beta$-escin, $38 \mu \mathrm{g} \mathrm{mL}^{-1}$ celecoxib, and $59 \mu \mathrm{g} \mathrm{mL}^{-1}$ pentamidine.) Screens were performed in duplicate using C. albicans, inoculated from YPD plates to YPD broth and cultured overnight at $30{ }^{\circ} \mathrm{C}, 120 \mathrm{rev} . \mathrm{min}^{-1}$.
Overnight cultures were diluted to $\mathrm{OD}_{600} 0.5$ and cultured for a further $4 \mathrm{~h}$ in fresh YPD before dilution of these experimental cultures to $\mathrm{OD}_{600} 0.01$ in the same medium. Aliquots $(100 \mu \mathrm{L})$ of the diluted culture plus any chemical supplements (see above) were transferred to 96-well plates (Greiner Bio-One) and cultured for $24 \mathrm{~h}$ at $30{ }^{\circ} \mathrm{C}, 120 \mathrm{rev}$. $\mathrm{min}^{-1}$, with $\mathrm{OD}_{600}$ measured at $24 \mathrm{~h}$ in a BioTek EL800 microplate spectrophotometer. $\mathrm{OD}_{600}$ was expressed as percentage of growth relative to control growth (i.e., without any chemicals). Effect strength [(\% growth with PL agent) - (\% growth with PL agent + second agent)] was calculated for each combination; screen hits were considered as those agents showing an effect strength $>50$.

Growth Inhibition, Toxicity, and Checkerboard Assays. General culturing and preparation for checkerboard assays adhered to EUCAST guidelines, ${ }^{37}$ with the exception that YPD broth was used here to validate the data from the screens. Briefly, either yeast cells from single colonies or A. fumigatus spores were inoculated from 2-d PDA plates to YPD broth adjusted to a final concentration of $10^{5}$ cells or spores $\mathrm{mL}^{-1}$. Culture aliquots $(100 \mu \mathrm{L})$ were transferred to 96 well microtiter plates with chemicals added at the specified concentrations. The inoculated plates were incubated statically for 24 (C. albicans and C. glabrata) or 48 (C. neoformans and A. fumigatus) hours at $30{ }^{\circ} \mathrm{C}$ (yeasts) or $37^{\circ} \mathrm{C}$ (A. fumigatus). $\mathrm{OD}_{600}$ was then measured with a BioTek EL800 microplate spectrophotometer. Fractional inhibitory concentration index (FICI) was calculated as an indicator of synergy. ${ }^{38}$ Assays for fungicidal or fungistatic activity were as described previously. ${ }^{22}$ Immortalized NIH 3T3 mouse embryonic fibroblast and human TE671 (rhabdomyosarcoma RD cell line) cells (passage 25) were cultured and toxicity to the cells was assayed (using the CCK- 8 reagent; Sigma) as described previously. ${ }^{13}$ All the assays were performed in independent triplicates.

Biofilm Inhibition Assays. Biofilm metabolic activity was measured by the XTT (tetrazolium salt, 2,3-bis[2-methyloxy-4nitro-5-sulfophenyl]-2H-tetrazolium-5-carboxanilide) (Sigma) reduction assay and performed as described previously. ${ }^{18,22}$ Briefly, overnight C. albicans cultures were diluted to $\mathrm{OD}_{600} \sim$ 0.01 in RPMI 1640 medium and $100 \mu \mathrm{L}$ aliquots transferred to 96-well microtiter plates (Greiner Bio-One). After removing nonadherent cells, yeasts were incubated at $37^{\circ} \mathrm{C}$ for $24 \mathrm{~h}$ in fresh medium. Biofilms were then washed with PBS and drugs added as specified. Cultures were incubated for a further $24 \mathrm{~h}$, then the biofilm was washed and the XTT reaction performed using $210 \mu \mathrm{g} \mathrm{mL}^{-1} \mathrm{XTT}$ and $4.2 \mu \mathrm{M}$ menadione. Biofilm metabolic activity was measured after $3 \mathrm{~h}$ at $490 \mathrm{~nm}$ using a BioTek El800 microplate spectrophotometer. The assays were performed in independent triplicates.

Mistranslation Assay. For quantitative determination of mistranslation, S. cerevisiae BY4743 was transformed using the lithium acetate method $^{39}$ with a dual luciferase reporter plasmid encoding firefly and Renilla luciferases either separated by a UAA stop codon ${ }^{40}$ or containing a missense codon in the ORF encoding firefly luciferase (His245 $\rightarrow \operatorname{Arg} 245)^{41}$ (kindly provided by D. Bedwell, University of Alabama, USA) as well as with a dual fluorescence reporter plasmid encoding GFP and RFP in frame (kindly provided by N. Altamura, Institute of Biomembranes and Bioenergetics, National Researches Council, Bari, Italy) ${ }^{42}$ and containing a missense codon in the ORF encoding GFP (Tyr66 $\rightarrow$ His66). In the latter case, the missense codon was introduced here by replacing Tyr66 with a 
histidine codon by site-directed mutagenesis, using the Q5 kit (New England BioLabs) with a plasmid containing the GFP and RFP separated by a CAA sense codon ${ }^{42}$ as DNA template. Precultures were prepared as described previously. ${ }^{13}$ Then, the cultures were diluted to $\mathrm{OD}_{600} 0.01$ in YNB medium, $100 \mu \mathrm{L}$ aliquots were transferred to 96-well microtiter plates, and the drugs were added as specified in the Results. Plates were incubated at $30{ }^{\circ} \mathrm{C}$ for $16 \mathrm{~h}$ with shaking in a BioTek Epoch 2 microplate spectrophotometer. For the dual luciferase assays, cell extracts were prepared and luciferase activities were measured as described previously. ${ }^{13}$ For the dual fluorescence assays, cells were harvested by centrifugation and resuspended in $500 \mu \mathrm{L}$ PBS before analysis of GFP and RFP fluorescence with a Beckman Coulter Astrios cytometer equipped with 488 and $561 \mathrm{~nm}$ lasers. The measurements of mistranslation rate were done in independent triplicates.

Assay of Paromomycin Uptake. To measure PM uptake, the aminoglycoside was conjugated to SFX [6-(fluorescein-5[and-6]-carboxamido) hexanoic acid, succinimidyl ester] (Invitrogen). Parmomycin sulfate salt $(100 \mathrm{mg}, 0.14 \mathrm{mmol})$ was dissolved in a $6 \mathrm{~mL}$ solution of $\mathrm{H}_{2} \mathrm{O}: \mathrm{MeOH}(2: 1$ ratio, v/ $\mathrm{v})$. Triethylamine $(100 \mu \mathrm{L})$ was added to this solution dropwise and stirred for $12 \mathrm{~h}$. SFX $(8 \mathrm{mg}, 0.014 \mathrm{mmol})$ dissolved in $\mathrm{MeOH}(0.5 \mathrm{~mL})$ was added dropwise over $15 \mathrm{~min}$ at $0{ }^{\circ} \mathrm{C}$ to a solution comprising neutralized paramomycin $(8$ $\mathrm{mg}, 0.013 \mathrm{mmol}$ ) and $10 \mu \mathrm{L}$ triethylamine dissolved in $\mathrm{H}_{2} \mathrm{O}: \mathrm{MeOH}(4: 1$ ratio, v/v). The reaction mixture was stirred for 2 days and then unreacted dye and trimethylamine were removed by dialysis for 1 day against water using a $1 \mathrm{kDa}$ molecular weight cutoff membrane. The purity of the final product was confirmed to be $\geq 97 \%$ by HPLC (Figure S1). Precultures and cultures in triplicate of $S$. cerevisiae were prepared as described above. Aliquots $(10 \mu \mathrm{L})$ of cell suspension $\left(\mathrm{OD}_{600} \sim 2.0\right)$ were incubated with PM-SFX \pm $\beta$-escin for $20 \mathrm{~min}$, then washed and resuspended in $500 \mu \mathrm{L}$ PBS before fluorescence from cellular PM-SFX was measured with a Beckman Coulter FC500 cytometer equipped with a $488 \mathrm{~nm}$ laser.

\section{ASSOCIATED CONTENT}

\section{SI Supporting Information}

The Supporting Information is available free of charge at https://pubs.acs.org/doi/10.1021/acsinfecdis.0c00405.

Figures S1, S2, S3 (PDF)

Table S1 (XLSX)

\section{AUTHOR INFORMATION}

\section{Corresponding Author}

Simon V. Avery - School of Life Sciences, University of Nottingham, Nottingham NG7 2RD, U.K.; 1 orcid.org/00000002-2102-2255; Phone: +44 (0)115 9513315;

Email: simon.avery@nottingham.ac.uk

\section{Authors}

Cindy Vallières - School of Life Sciences, University of Nottingham, Nottingham NG7 2RD, U.K.

Nishant Singh - School of Pharmacy, University of Nottingham, Nottingham NG7 2RD, U.K.

Cameron Alexander - School of Pharmacy, University of Nottingham, Nottingham NG7 2RD, U.K.; 10 orcid.org/00000001-8337-1875

Complete contact information is available at: https://pubs.acs.org/10.1021/acsinfecdis.0c00405

\section{Funding}

This work was supported by the Biotechnology and Biological Sciences Research Council (Grant Number BB/P02369X/1).

\section{Notes}

The authors declare no competing financial interest.

\section{REFERENCES}

(1) Brown, G. D., Denning, D. W., and Levitz, S. M. (2012) Tackling human fungal infections. Science 336, 647.

(2) Fisher, M. C., Hawkins, N. J., Sanglard, D., and Gurr, S. J. (2018) Worldwide emergence of resistance to antifungal drugs challenges human health and food security. Science 360, 739-742.

(3) Roemer, T., and Krysan, D. J. (2014) Antifungal drug development: challenges, unmet clinical needs, and new approaches. Cold Spring Harbor Perspect. Med. 4, a019703.

(4) Patil, A., and Majumdar, S. (2017) Echinocandins in antifungal pharmacotherapy. J. Pharm. Pharmacol. 69, 1635-1660.

(5) Johnson, M. D., MacDougall, C., Ostrosky-Zeichner, L., Perfect, J. R., and Rex, J. H. (2004) Combination antifungal therapy. Antimicrob. Agents Chemother. 48, 693-715.

(6) Zimmermann, G. R., Lehar, J., and Keith, C. T. (2007) Multitarget therapeutics: when the whole is greater than the sum of the parts. Drug Discovery Today 12, 34-42.

(7) Spitzer, M., Robbins, N., and Wright, G. D. (2017) Combinatorial strategies for combating invasive fungal infections. Virulence 8, 169-185.

(8) Dromer, F., Bernede-Bauduin, C., Guillemot, D., and Lortholary, O. (2008) French Cryptococcosis Study G. Major role for amphotericin B-flucytosine combination in severe cryptococcosis. PLoS One 3, e2870.

(9) Spitzer, M., Griffiths, E., Blakely, K. M., Wildenhain, J., Ejim, L., Rossi, L., De Pascale, G., Curak, J., Brown, E., Tyers, M., and Wright, G. D. (2011) Cross-species discovery of syncretic drug combinations that potentiate the antifungal fluconazole. Mol. Syst. Biol. 7, 499.

(10) Robbins, N., Spitzer, M., Yu, T., Cerone, R. P., Averette, A. K., Bahn, Y. S., Heitman, J., Sheppard, D. C., Tyers, M., and Wright, G. D. (2015) An antifungal combination matrix identifies a rich pool of adjuvant molecules that enhance drug activity against diverse fungal pathogens. Cell Rep. 13, 1481-1492.

(11) Holbrook, S. Y. L., Garzan, A., Dennis, E. K., Shrestha, S. K., and Garneau-Tsodikova, S. (2017) Repurposing antipsychotic drugs into antifungal agents: Synergistic combinations of azoles and bromperidol derivatives in the treatment of various fungal infections. Eur. J. Med. Chem. 139, 12-21.

(12) Kim, J. H., Chan, K. L., Cheng, L. W., Tell, L. A., Byrne, B. A., Clothier, K., and Land, K. M. (2019) High efficiency drug repurposing design for new antifungal agents. Methods Protoc 2, 31.

(13) Moreno-Martinez, E., Vallieres, C., Holland, S. L., and Avery, S. V. (2015) Novel, synergistic antifungal combinations that target translation fidelity. Sci. Rep. 5, 16700.

(14) Cederlund, H. J., and Mardh, P. A. (1993) Antimicrobial activities of $\mathrm{N}$-acetylcysteine and some non-steroidal antiinflammatory drugs. J. Antimicrob. Chemother. 32, 903-904.

(15) Laleve, A., Vallieres, C., Golinelli-Cohen, M. P., Bouton, C., Song, Z., Pawlik, G., Tindall, S. M., Avery, S. V., Clain, J., and Meunier, B. (2016) The antimalarial drug primaquine targets Fe-S cluster proteins and yeast respiratory growth. Redox Biol. 7, 21-29.

(16) Anderson, J. B. (2005) Evolution of antifungal-drug resistance: mechanisms and pathogen fitness. Nat. Rev. Microbiol. 3, 547-556.

(17) Cavalheiro, M., and Teixeira, M. C. (2018) Candida biofilms: threats, challenges, and promising strategies. Front. Med. 5, 28.

(18) Vallieres, C., Hook, A. L., He, Y., Cuzzucoli Crucitti, V., Figueredo, G., Davies, C. R., Burroughs, L., Winkler, D. A., Wildman, R. D., Irvine, D. J., Alexander, M. R., and Avery, S. V. (2020) Discovery of (meth)acrylate polymers that resist colonization by fungi 
associated with pathogenesis and biodeterioration. Science Adv. 6, eaba6574.

(19) Omelkova, J., Slavikova, E., and Vadkerti, A. (1990) Inhibiting effect of betaescine on the growth of yeasts and yeast microorganism. Biologia 45, 925-929.

(20) Franiczek, R., Glensk, M., Krzyzanowska, B., and Wlodarczyk, M. (2015) beta-Aescin at subinhibitory concentration (sub-MIC) enhances susceptibility of Candida glabrata clinical isolates to nystatin. Med. Mycol. 53, 845-851.

(21) Morton, M. J., and Main, M. J. (2013) Use of escin as a perforating agent on the IonWorks quattro automated electrophysiology platform. J. Biomol. Screening 18, 128-134.

(22) Vallieres, C., Raulo, R., Dickinson, M., and Avery, S. V. (2018) Novel combinations of agents targeting translation that synergistically inhibit fungal pathogens. Front. Microbiol. 9, 2355.

(23) Campbell, B. C., Chan, K. L., and Kim, J. H. (2012) Chemosensitization as a means to augment commercial antifungal agents. Front. Microbiol. 3, 79.

(24) Cokol, M., Chua, H. N., Tasan, M., Mutlu, B., Weinstein, Z. B., Suzuki, Y., Nergiz, M. E., Costanzo, M., Baryshnikova, A., Giaever, G., Nislow, C., Myers, C. L., Andrews, B. J., Boone, C., and Roth, F. P. (2011) Systematic exploration of synergistic drug pairs. Mol. Syst. Biol. 7,544 .

(25) Vallieres, C., and Avery, S. V. (2017) The candidate antimalarial drug MMV665909 causes oxygen-dependent mRNA mistranslation and synergizes with quinoline-derived antimalarials. Antimicrob. Agents Chemother. 61, e0045917.

(26) Daxboeck, F., Rabitsch, W., Stadler, M., Assadian, O., and Leitgeb, J. (2013) High resistance of Pseudomonas aeruginosa to paromomycin, an agent used for selective bowel decontamination (SBD). GMS Hyg. Infect. Control 8, Doc04.

(27) Musa, A. M., Younis, B., Fadlalla, A., Royce, C., Balasegaram, M., Wasunna, M., Hailu, A., Edwards, T., Omollo, R., Mudawi, M., Kokwaro, G., El-Hassan, A., and Khalil, E. (2010) Paromomycin for the treatment of visceral leishmaniasis in Sudan: a randomized, openlabel, dose-finding study. PLoS Neglected Trop. Dis. 4, e855.

(28) Mutyam, V., Du, M., Xue, X., Keeling, K. M., White, E. L., Bostwick, J. R., Rasmussen, L., Liu, B., Mazur, M., Hong, J. S., Falk Libby, E., Liang, F., Shang, H., Mense, M., Suto, M. J., Bedwell, D. M., and Rowe, S. M. (2016) Discovery of clinically approved agents that promote suppression of cystic fibrosis transmembrane conductance regulator nonsense mutations. Am. J. Respir. Crit. Care Med. 194, $1092-1103$.

(29) Ng, M. Y., Zhang, H., Weil, A., Singh, V., Jamiolkowski, R., Baradaran-Heravi, A., Roberge, M., Jacobson, A., Friesen, W., Welch, E., Goldman, Y. E., and Cooperman, B. S. (2018) New in vitro assay measuring direct interaction of nonsense suppressors with the eukaryotic protein synthesis machinery. ACS Med. Chem. Lett. 9, 1285-1291.

(30) Koselny, K., Green, J., DiDone, L., Halterman, J. P., Fothergill, A. W., Wiederhold, N. P., Patterson, T. F., Cushion, M. T., Rappelye, C., Wellington, M., and Krysan, D. J. (2016) The celecoxib derivative AR-12 has broad-spectrum antifungal activity in vitro and improves the activity of fluconazole in a murine model of cryptococcosis. Antimicrob. Agents Chemother. 60, 7115-7127.

(31) Koselny, K., Green, J., Favazzo, L., Glazier, V. E., DiDone, L., Ransford, S., and Krysan, D. J. (2016) Antitumor/antifungal celecoxib derivative AR-12 is a non-nucleoside inhibitor of the ANL-family adenylating enzyme acetyl CoA synthetase. ACS Infect. Dis. 2, 268280.

(32) Tiwari, S., Mishra, M., Salemi, M. R., Phinney, B. S., Newens, J. L., and Gomes, A. V. (2020) Gender-specific changes in energy metabolism and protein degradation as major pathways affected in livers of mice treated with ibuprofen. Sci. Rep. 10, 3386.

(33) Panozzo, C., Laleve, A., Tribouillard-Tanvier, D., Ostojic, J., Sellem, C. H., Friocourt, G., Bourand-Plantefol, A., Burg, A., Delahodde, A., Blondel, M., and Dujardin, G. (2017) Chemicals or mutations that target mitochondrial translation can rescue the respiratory deficiency of yeast bcs1 mutants. Biochim. Biophys. Acta, Mol. Cell Res. 1864, 2297-2307.

(34) Brent, K. J., and Hollomon, D. W. (2007) Fungicide Resistance in Crop Pathogens: How Can It Be Managed?, Fungicide Resistance Action Committee, Belgium.

(35) Hill, J. A., O’Meara, T. R., and Cowen, L. E. (2015) Fitness trade-offs associated with the evolution of resistance to antifungal drug combinations. Cell Rep. 10, 809-819.

(36) Ausubel, F. M., Brent, R., Kingston, R. E., Moore, D. D., Seidman, J. G., and Struhl, K. (2007) Current Protocols in Molecular Biology, John Wiley and Sons, New York.

(37) Arendrup, M. C., Meletiadis, J., Mouton, J. W., Lagrou, K., Hamal, P., and Guinea, J., and the Subcommittee on Antifungal Susceptibility Testing (AFST) of the ESCMID European Committee for Antimicrobial Susceptibility Testing; (2017) Method for the determination of broth dilution minimum inhibitory concentrations of antifungal agents for yeasts. EUCAST Antifungal MIC Method for Yeasts, Vol. 7.3.1, pp 1-21.

(38) Hsieh, M. H., Yu, C. M., Yu, V. L., and Chow, J. W. (1993) Synergy assessed by checkerboard. A critical analysis. Diagn. Microbiol. Infect. Dis. 16, 343-349.

(39) Gietz, R. D., and Woods, R. A. (2002) Transformation of yeast by lithium acetate/single-stranded carrier DNA/polyethylene glycol method. Methods Enzymol. 350, 87-96.

(40) Keeling, K. M., Lanier, J., Du, M., Salas-Marco, J., Gao, L., Kaenjak-Angeletti, A., and Bedwell, D. M. (2004) Leaky termination at premature stop codons antagonizes nonsense-mediated mRNA decay in S. cerevisiae. RNA 10, 691-703.

(41) Salas-Marco, J., and Bedwell, D. M. (2005) Discrimination between defects in elongation fidelity and termination efficiency provides mechanistic insights into translational readthrough. J. Mol. Biol. 348, 801-815.

(42) Altamura, E., Borgatti, M., Finotti, A., Gasparello, J., Gambari, R., Spinelli, M., Castaldo, R., and Altamura, N. (2016) Chemicalinduced read-through at premature termination codons determined by a rapid dual-fluorescence system based on S. cerevisiae. PLoS One 11, e0154260. 